\title{
SOME PROPERTIES OF CERTAIN MEROMORPHIC MULTIVALENT CLOSE-TO-CONVEX FUNCTIONS
}

\author{
HONG-GUANG LI
}

Received 18 October, 2019

\begin{abstract}
In this paper, we introduce and investigate a certain subclass of meromorphic multivalent close-to-convex functions. Such results as coefficient inequalities, and radius of meromorphic convexity are derived.
\end{abstract}

2010 Mathematics Subject Classification: 30C55; 28A80

Keywords: meromorphic close-to-convex function, multivalent function,convexity

\section{INTRODUCTION}

Let $\sum_{p}$ denote the class of functions $\mathrm{f}$ of the form

$$
f(z)=\frac{1}{z^{p}}+\sum_{n=0}^{\infty} a_{n} z^{n}(p \in \mathbb{N}:=\{1,2,3 \cdots\}),
$$

which are analytic in the punctured open unit disk

$$
\mathbb{U}^{*}:=\{z: z \in \mathbb{C} \text { and } 0<|z|<1\}=: \mathbb{U} \backslash 0 .
$$

Let $\mathcal{P}$ denote the class of functions $\mathrm{p}$ given by

$$
p(z)=1+\sum_{n=1}^{\infty} b_{n} z^{n}(z \in \mathbb{U}),
$$

which are analytic and convex in $\mathbb{U}$ and satisfy the condition $\mathbb{R}(p(z))>0(z \in \mathbb{U})$.

A function $f \in \sum_{p}$ is said to be in the class $\mathcal{M} S_{p}^{*}(\alpha)$ of meromorphic multivalent starlike functions of order $\alpha$ if it satisfies the inequality

$$
\mathbb{R}\left(\frac{z f^{\prime}(z)}{f(z)}\right)<-\alpha(z \in \mathbb{U}, 0 \leq \alpha<p) .
$$

The author was supported by the NNSF of China, No.11831007. 
Let

$$
g(z)=\frac{1}{z^{p}}+\sum_{n=0}^{\infty} c_{n} z^{n} \in \mathcal{M} S_{p}^{*}(\alpha),
$$

a function $f \in \sum_{p}$ is said to be in the class $\mathcal{M} C_{p}$ of meromorphic multivalent closeto-convex functions if it satisfies the inequality

$$
\mathbb{R}\left(\frac{z f^{\prime}(z)}{g(z)}\right)<0\left(z \in \mathbb{U}, g \in \mathcal{M} S_{p}^{*}(0)=: \mathcal{M} S_{p}^{*}\right) .
$$

In many earlier investigations(for example [2,3,5-7,10-12,15-21,24]), various interesting subclasses of the close-to-convex functions have been studied from a number of different viewpoints. In particular, Gao and Zhou[3](see also [7, 10, 21, 24]) considered a subclass $\mathcal{K}_{s}$ of close-to-convex functions, which satisfy the condition

$$
\mathbb{R}\left(\frac{z^{2} f^{\prime}(z)}{g(z) g(-z)}\right)<0\left(z \in \mathbb{U}, g \in \mathcal{S}^{*}(1 / 2)\right),
$$

where $f(z)=z+a_{2} z^{2}+\cdots$, and $\mathcal{S}^{*}(1 / 2)$ denotes the usual class of starlike functions of order $1 / 2$.

Recently, Z.G.Wang et al.[22] introduced the meromophic close-to-convex functions class $\mathcal{M} \mathcal{K}$, which satisfy the condition

$$
\mathbb{R}\left(\frac{f^{\prime}(z)}{g(z) g(-z)}\right)>0\left(z \in \mathbb{U}, g \in \mathcal{M S}^{*}(p / 2)\right),
$$

where $f(z)=1 / z+a_{1} z+a_{2} z^{2}+\cdots$.

Motivated essentially by the above mentioned works, we introduce a class of meoromorphic multivalent functions related to the meoromorphic multivalent starlike functions, and obtain some interesting results.

Definition 1. A function $f \in \sum_{p}$ is said to be in the $\mathcal{M} \mathcal{K}_{p}$ if it satisfies the inequality

$$
\mathbb{R}\left(\frac{z f^{\prime}(z)}{z^{p} g(z) g(-z)}\right)>0(z \in \mathbb{U})
$$

where $g \in \mathcal{M} S_{p}^{*}(p / 2)$.

For some recent investigation of meromorphic multivalent functions, see (for example)the works of $[1,8,9,13,23,25]$ and the references cited therein.

In the present paper, we prove that the class $\mathcal{M} \mathcal{K}_{p}$ is a subclass of meromorphic multivalent close-to-convex functions.

Theorem 1. Suppose that $\mu(z) \in \mathcal{M} S_{p}^{*}\left(\alpha_{1}\right)$ and $v(z) \in \mathcal{M} S_{p}^{*}\left(\alpha_{2}\right)$ with $0 \leq \alpha_{1}+$ $\alpha_{2}-p<p$. Then

$$
z^{p} \mu(z) v(z) \in \mathcal{M} S_{p}^{*}\left(\alpha_{1}+\alpha_{2}-p\right)
$$


Proof of Theorem 1. Let $\mu(z) \in \mathcal{M} S_{p}^{*}\left(\alpha_{1}\right)$ and $v(z) \in \mathcal{M} S_{p}^{*}\left(\alpha_{2}\right)$. By definition, we know that

$$
\mathbb{R}\left(\frac{z \mu^{\prime}(z)}{\mu(z)}\right)<-\alpha_{1}\left(z \in \mathbb{U}, 0 \leq \alpha_{1}<p\right),
$$

and

$$
\mathbb{R}\left(\frac{z v^{\prime}(z)}{v(z)}\right)<-\alpha_{2}\left(z \in \mathbb{U}, 0 \leq \alpha_{2}<p\right) .
$$

Next, we assume that

$$
h(z)=z^{p} \mu(z) v(z) .
$$

Then, we easily get

$$
\frac{z h^{\prime}(z)}{h(z)}=\frac{z \mu^{\prime}(z)}{\mu(z)}+\frac{z v^{\prime}(z)}{v(z)}+p
$$

It follows that

$$
\mathbb{R}\left(\frac{z h^{\prime}(z)}{h(z)}\right)=\mathbb{R}\left(\frac{z \mu^{\prime}(z)}{\mu(z)}\right)+\mathbb{R}\left(\frac{z v^{\prime}(z)}{v(z)}\right)+p<-\left(\alpha_{1}+\alpha_{2}-p\right) .
$$

Noting that $0 \leq \alpha_{1}+\alpha_{2}-p<p$, which implies that

$$
h(z) \in \mathcal{M} S_{p}^{*}\left(\alpha_{1}+\alpha_{2}-p\right) .
$$

This completes the proof of Theorem 1.

Theorem 2. Let $g \in \mathcal{M} S_{p}^{*}(p / 2)$. Then

$$
-z^{p} g(z) g(-z) \in \mathcal{M} S_{p}^{*}(0)=: \mathcal{M} S_{p}^{*} .
$$

Proof of Theorem 2. Similar to the proof of Theorem 1, we can get

$$
\mathbb{R}\left(\frac{z\left(-z^{p} g(z) g(-z)\right)^{\prime}}{-z^{p} g(z) g(-z)}\right)=p+\mathbb{R}\left(\frac{z g^{\prime}(z)}{g(z)}\right)+\mathbb{R}\left(\frac{-z g^{\prime}(-z)}{g(-z)}\right)<p-\frac{p}{2}-\frac{p}{2}=0
$$

(noting that $-z \in \mathbb{U}$ ). This implies the Theorem 2.

In view of the definitions $\mathcal{M} \mathcal{C}_{p}, \mathcal{M} \mathcal{K}_{p}$ and Theorem 2, we deduce that the class $\mathscr{M} \mathcal{K}_{p}$ is a subclass of the class $\mathscr{M} C_{p}$ of meromorphic close-to-convex functions.

To derive coefficient inequalities of $f \in \mathcal{M} \mathcal{K}_{p}$, we need consider the parity of $p$. First, we consider the case that $p$ is odd. 


\section{THE CASE $\mathrm{P}=2 \mathrm{~K}-1$}

In order to prove our main results, we need the following two lemmas.

Lemma 1 ([14]). Suppose that $h(z)=\frac{1}{z^{p}}+\sum_{n=0}^{\infty} c_{n} z^{n} \in \mathcal{M} S_{p}^{*}$. Then

$$
\left|c_{n}\right| \leq(2 p) /(n+p)(n \in \mathbb{N}:=\{1,2,3 \cdots\}) .
$$

Equality holds for the function $h(z)=z^{-p}\left(1+z^{n+p}\right)^{2 p /(n+p)}$.

Lemma 2. Let $g(z)=\frac{1}{z^{p}}+\sum_{n=0}^{\infty} b_{n} z^{n} \in \mathcal{M} S_{p}^{*}(p / 2)$. Then

$$
\left|B_{2 m-1}\right| \leq(2 p) /(2 m-1+p)(m \in \mathbb{N}),
$$

where

$$
B_{2 m-1}= \begin{cases}2 b_{2 m-1}, & 2 m-1<p, \\ 2 b_{2 m-1}-2 b_{0} b_{2 m-1-p}+2 b_{1} b_{2 m-2-p}-\cdots+ & \\ (-1)^{m-\frac{p+1}{2}} 2 b_{m-\frac{p+3}{2}} b_{m-\frac{p-1}{2}}+(-1)^{m-\frac{p-1}{2}} b_{m-\frac{p+1}{2}}^{2}, & 2 m-1 \geq p .\end{cases}
$$

Equality holds for the function $g(z)=z^{-p}\left(1+z^{n+p}\right)^{p /(n+p)}$.

Proof. Suppose that

$$
G(z):=-z^{p} g(z) g(-z) .
$$

In view of Theorem 2 , we know that $G(z) \in \mathcal{M} S_{p}^{*}$. When $p=2 k-1(k=1,2, \cdots)$, it is easy to verify that

$$
G(-z)=-G(z)
$$

which implies that $G(z)$ is a meromorphic odd starlike multivalent function. If we set

$$
G(z)=\frac{1}{z^{p}}+\sum_{m=1}^{\infty} B_{2 m-1} z^{2 m-1},
$$

it follows from Lemma 1 that

$$
\left|B_{2 m-1}\right| \leq(2 p) /(2 m-1+p)(m \in \mathbb{N}) .
$$

By substituting the series expressions of $g(z)$ and $G(z)$ into (2.2) and carefully comparing the similar items of two sides of resulting equation, we get the desired expression of $B_{2 m-1}$ given by (2.1).

Theorem 3. Suppose that $f(z)=\frac{1}{z^{p}}+\sum_{n=0}^{\infty} a_{n} z^{n} \in \mathcal{M} \mathcal{K}_{p}$. Then

$$
\left|a_{2 n}\right| \leq\left\{\begin{array}{ll}
p / n, & 2 n-3<p, \\
\frac{p}{n}\left(1+\frac{2 p}{n-1}+\frac{2 p}{n-2}+\cdots+\frac{2 p}{1+p}\right), & 2 n-3 \geq p,
\end{array}(n \in \mathbb{N})\right.
$$


and

$$
(2 n-1)\left|a_{2 n-1}\right| \leq \begin{cases}2 p+\frac{2 p^{2}}{(2 n-1+p)}, & 2 n-3<p, \\ 2 p+\frac{2 p^{2}}{n-1}+\frac{2 p^{2}}{n-2}+\cdots+\frac{2 p^{2}}{1+p}+\frac{2 p^{2}}{2 n-1+p}, & 2 n-3 \geq p .\end{cases}
$$

Proof of Theorem 3. Suppose that $f \in \mathcal{M} \mathcal{K}_{p}$. Then, we know that $\mathbb{R}\left(\frac{z f^{\prime}(z)}{G(z)}\right)<0$, where $G$ is given by (2.2). If we set

$$
q(z):=-\frac{z f^{\prime}(z)}{p G(z)}
$$

it follows that

$$
q(z)=1+d_{p+1} z^{p+1}+d_{p+2} z^{p+2}+\cdots \in \mathcal{P} .
$$

By substituting the series expressions of $f, G$ and $q$ into (2.7), we get

$$
\begin{gathered}
p\left(1+d_{p+1} z^{p+1}+\cdots+d_{p+n} z^{p+n}+\cdots\right)\left(\frac{1}{z^{p}}+B_{1} z+B_{3} z^{3}+\cdots+B_{2 n-1} z^{2 n-1}+\cdots\right) \\
=\frac{p}{z^{p}}-a_{1} z-2 a_{2} z^{2}-\cdots-2 n a_{2 n} z^{2 n}-(2 n+1) a_{2 n+1} z^{2 n+1}-\cdots
\end{gathered}
$$

We get from (2.8) that

$$
\frac{-2 n}{p} a_{2 n}= \begin{cases}d_{p+2 n}, & 2 n-3<p, \\ d_{p+2 n}+d_{p+2} B_{2 n-2-p}+\cdots+d_{2 n-1} B_{1}, & 2 n-3 \geq p,\end{cases}
$$

and

$$
\frac{2 n-1}{-p} a_{2 n-1}= \begin{cases}d_{p+2 n-1}+B_{2 n-1}, & 2 n-3<p, \\ d_{p+2 n-1}+B_{2 n-1}+d_{p+1} B_{2 n-p-2} & \\ +d_{p+2} B_{2 n-p-3}+\cdots+d_{2 n-2} B_{1}, & 2 n-3 \geq p .\end{cases}
$$

For $q(z) \in \mathcal{P}$, we know that $\left|d_{n+p}\right| \leq 2$ ([4]). Moreover, combining (2.4), ( 2.8), (2.9) and (2.10), we get (2.5) and (2.6).

Theorem 4. Let $g(z)=\frac{1}{z^{p}}+\sum_{n=0}^{\infty} b_{n} z^{n} \in \mathcal{M} S_{p}^{*}(p / 2)$. If $f \in \sum_{p}$ satisfies condition

$$
\sum_{n=1}^{\infty} n\left|a_{n}\right|+p \sum_{n=1}^{\infty}\left|B_{2 n-1}\right| \leq p
$$

where $B_{2 n-1}$ is given by (2.1), then $f \in \mathcal{M} \mathcal{K}_{p}$.

Proof of Theorem 4. To prove $f \in \mathcal{M} \mathcal{K}_{p}$, it needs to show that

$$
\mathbb{R}\left(\frac{f^{\prime}(z)}{g(z) g(-z)}\right)=\mathbb{R}\left(\frac{z f^{\prime}(z)}{G(z)}\right)>0,
$$


i,e, it suffices to show that

$$
\left|\frac{z f^{\prime}(z)}{G(z)}+p\right|<\left|\frac{z f^{\prime}(z)}{G(z)}-p\right|
$$

where $G$ is given by (2.3). From (2.11), it is easy to know that

$$
\sum_{n=1}^{\infty} n\left|a_{n}\right|+p \sum_{n=1}^{\infty}\left|B_{2 n-1}\right| \leq 2 p-\sum_{n=1}^{\infty} n\left|a_{n}\right|-p \sum_{n=1}^{\infty}\left|B_{2 n-1}\right| .
$$

Now, by the maximum principle, we deduce from (1.1) and (2.12) that

$$
\begin{aligned}
& \left|\frac{\frac{z f^{\prime}(z)}{G(z)}+p}{\frac{z f^{\prime}(z)}{G(z)}-p}\right|=\left|\frac{\sum_{n=1}^{\infty} n a_{n} z^{n+p}+\sum_{n=1}^{\infty} p B_{2 n-1} z^{2 n+p-1}}{\sum_{n=1}^{\infty} n a_{n} z^{n+p}-\sum_{n=1}^{\infty} p B_{2 n-1} z^{2 n+p-1}-2 p}\right| \\
& \quad<\frac{\sum_{n=1}^{\infty} n\left|a_{n}\right|+\sum_{n=1}^{\infty} p\left|B_{2 n-1}\right|}{2 p-\sum_{n=1}^{\infty} n\left|a_{n}\right|-\sum_{n=1}^{\infty} p\left|B_{2 n-1}\right|} \leq 1 .
\end{aligned}
$$

This evidently complete proof of Theorem 4.

Moreover, we consider the case that $p$ is even.

\section{THE CASE $\mathrm{P}=2 \mathrm{~K}$}

By similarly applying the method of proof of Lemma 3, we easily get the following Lemma.

Lemma 3. Let $p=2 k, k \in \mathbb{N}$ and

$$
g(z)=\frac{1}{z^{p}}+\sum_{n=0}^{\infty} b_{n} z^{n} \in \mathcal{M} S_{p}^{*}(p / 2) .
$$

Then

$$
\left|B_{2 m}\right| \leq(2 p) /(2 m+p)(m \in \mathbb{N}),
$$

where

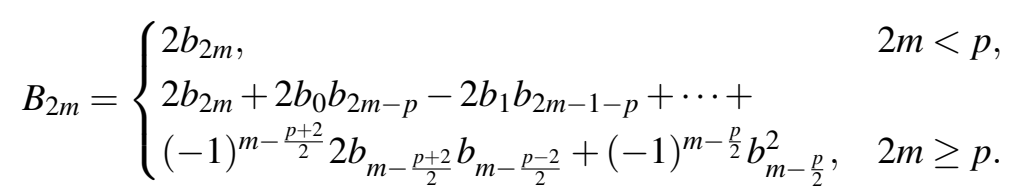

Equality holds for the function $h(z)=z^{-p}\left(1+z^{n+p}\right)^{p /(n+p)}$.

Theorem 5. Suppose that

$$
f(z)=\frac{1}{z^{p}}+\sum_{n=0}^{\infty} a_{n} z^{n} \in \mathcal{M} \mathcal{K}_{p} .
$$


Then

$$
\left|a_{2 n}\right| \leq\left\{\begin{array}{ll}
p / n+p^{2} /\left(2 n^{2}+n p\right), & 2 n<p, \\
\frac{p}{n}\left(1+\frac{2 p}{n-1}+\frac{2 p}{n-2}+\cdots+\frac{2 p}{1+p}+2\right)+\frac{p^{2}}{2 n^{2}+n p}, & 2 n \geq p,
\end{array}(n \in \mathbb{N})\right.
$$

and

$$
(2 n-1)\left|a_{2 n-1}\right| \leq \begin{cases}2 p, & 2 n-2<p \\ 2 p+\frac{2 p^{2}}{n-1}+\frac{2 p^{2}}{n-2}+\cdots+\frac{2 p^{2}}{1+p}, & 2 n-2 \geq p .\end{cases}
$$

Proof of Theorem 5. Suppose that $f \in \mathcal{M} \mathcal{K}_{\varphi}$. Then, we know that $\mathbb{R}\left(\frac{z f^{\prime}(z)}{G(z)}\right)<0$, where $G$ is given by (2.2). For $p=2 k, k \in \mathbb{N}$, it is easy to deduce that $G(z)$ is a meromorphic even starlike multivalent function. If we set

$$
G(z)=\frac{1}{z^{p}}+\sum_{m=0}^{\infty} B_{2 m} z^{2 m}
$$

where $B_{2 m}$ is defined by (3.2) and

$$
\tau(z):=-\frac{z f^{\prime}(z)}{p G(z)}
$$

it follows that

$$
\tau(z)=1+d_{p} z^{p}+d_{p+1} z^{p+1}+\cdots \in \mathcal{P} .
$$

By substituting the series expressions of $f, G$ and $\tau$ into (3.6), we get

$$
\begin{gathered}
p\left(1+d_{p} z^{p}+d_{p+1} z^{p+1}+\cdots+d_{p+n} z^{p+n}+\cdots\right)\left(\frac{1}{z^{p}}+B_{0}+B_{2} z^{2}+\cdots+B_{2 n} z^{2 n}+\cdots\right) \\
=\frac{p}{z^{p}}-a_{1} z-2 a_{2} z^{2}-\cdots-2 n a_{2 n} z^{2 n}-(2 n+1) a_{2 n+1} z^{2 n+1}-\cdots
\end{gathered}
$$

We get from (3.7) that

$$
\frac{-2 n}{p} a_{2 n}= \begin{cases}d_{p+2 n}+B_{2 n}, & 2 n<p \\ d_{p+2 n}+B_{2 n}+d_{p+2} B_{2 n-2-p}+\cdots+d_{2 n} B_{0}, & 2 n \geq p\end{cases}
$$

and

$$
\frac{2 n-1}{-p} a_{2 n-1}= \begin{cases}d_{p+2 n-1}, & 2 n-2<p \\ d_{p+2 n-1}+d_{p+1} B_{2 n-p-2}+\cdots+d_{2 n-2} B_{1}, & 2 n-2 \geq p .\end{cases}
$$

For $\tau(z) \in \mathcal{P}$, we know that $\left|d_{n+p}\right| \leq 2$ (see [4]). Moreover, combining (3.1),(3.7),(3.8) and (3.9), we get (3.3) and (3.4).

Theorem 6. If $f \in \sum_{p}$ satisfies condition

$$
\sum_{n=1}^{\infty} n\left|a_{n}\right|+p \sum_{n=0}^{\infty}\left|B_{2 n}\right| \leq p
$$


where $B_{2 n}$ is given by (3.2), then $f \in \mathcal{M} \mathcal{K}_{p}$.

Proof of Theorem 6. The proof of Theorem 6 is similar to Theorem 4, we here omit the details.

\section{ON THE CONVEXITY RADIUS OF THE FUNCTIONS IN $\mathcal{M} \mathcal{K}_{p}$}

We say a function $f(z) \in \mathcal{M} \mathcal{K}_{p}$ is meromorphic convex, if $f(z)$ satisfies condition:

$$
\mathbb{R}\left(1+\frac{z f^{\prime \prime}(z)}{f^{\prime}(z)}\right)<0(z \in \mathbb{U}) .
$$

When we give the convexity radius of the functions in $\mathcal{M} \mathcal{K}_{p}$, we need the following lemmas.

Lemma 4. Let $G(z)$ is given by (2.2) and $r<1$, then

$$
\mathbb{R}\left(\frac{z G^{\prime}(z)}{G(z)}\right) \leq-\frac{1-r^{2}}{1+r^{2}} p(|z|=r) .
$$

Proof. Suppose that

$$
H(z):=-\frac{z G^{\prime}(z)}{p G(z)}\left(G(z) \in \mathcal{M} S_{p}^{*}\right),
$$

where $G(z)$ is given by (2.2), we easily know that $G(z)$ is an odd or even meromorphic starlike function, also $H(z) \in \mathcal{P}$ and is an even function, which imply that

$$
H(z)=\frac{1+[w(z)]^{2}}{1-[w(z)]^{2}}
$$

where $w(z)$ is Schwarz function with $w(0)=0$ and $|w(z)|<1$. Thus, we get from (4.2) that

$$
[w(z)]^{2}=\frac{H(z)-1}{H(z)+1}
$$

So

$$
\left|\frac{H(z)-1}{H(z)+1}\right|=|w(z)|^{2} \leq|z|^{2},
$$

this inequality can be written as

$$
|H(z)|^{2}-2 \operatorname{Re}\{H(z)\}+1 \leq|z|^{4}\left\{|H(z)|^{2}+2 \operatorname{Re}\{H(z)\}+1\right\} .
$$

From above inequality we can get

$$
\left|H(z)-\frac{1+|z|^{4}}{1-|z|^{4}}\right|^{2} \leq\left(\frac{1+|z|^{4}}{1-|z|^{4}}\right)^{2}-1 \leq\left(\frac{2|z|^{2}}{1-|z|^{4}}\right)^{2},
$$


that is

$$
\left|H(z)-\frac{1+|z|^{4}}{1-|z|^{4}}\right| \leq \frac{2|z|^{2}}{1-|z|^{4}} .
$$

From this inequality we get

$$
\mathbb{R}\{-H(z)\} \leq-\frac{1-|z|^{2}}{1+|z|^{2}}=-\frac{1-r^{2}}{1+r^{2}},
$$

this implies Lamma 4.

Lemma 5 (see [3]). Let $q(z)$ satisfy $q(0)=1, \mathbb{R}\{q(z)\}>0$, then we have

$$
\left|\frac{z q^{\prime}(z)}{q(z)}\right| \leq \frac{2 r}{1-r^{2}}(|z|=r<1)
$$

Theorem 7. Let $f(z) \in \mathcal{M} \mathcal{K}_{p}$, then $f(z)$ is meromorphic convex in

$$
0<|z|<r_{p}=\frac{1}{2} \sqrt{4+\frac{1}{p^{2}}}-\frac{\sqrt{\frac{1}{\sqrt{4+\frac{1}{p^{2}}} p^{3}}+\frac{1}{p^{2}}+\frac{4}{\sqrt{4+\frac{1}{p^{2}}} p}}}{\sqrt{2}}+\frac{1}{2 p} .
$$

Proof of Theorem 7. When $f(z) \in \mathcal{M} \mathcal{K}_{p}$, there exists $g(z) \in \mathcal{M} S_{p}^{*}(p / 2)$ such that ( 1.4) holds, also $G(z)=-z^{p} g(z) g(-z)$ is an odd or even meromophic starlike multivalent function, so from (2.7) and (3.5) we have

$$
z f^{\prime}(z)=-p G(z) \cdot q(z)
$$

where $q(z)$ satisfies the condition of Lemma 5 , and

$$
1+\frac{z f^{\prime \prime}(z)}{f^{\prime}(z)}=\frac{z G^{\prime}(z)}{G(z)}+\frac{z q^{\prime}(z)}{q(z)} .
$$

So using Lemma 4 and 5 we can get

$$
\begin{aligned}
\mathbb{R}\left\{1+\frac{z f^{\prime \prime}(z)}{f^{\prime}(z)}\right\}=\mathbb{R}\left\{\frac{z G^{\prime}(z)}{G(z)}\right\} & +\mathbb{R}\left\{\frac{z q^{\prime}(z)}{q(z)}\right\} \\
\leq & -\frac{1-r^{2}}{1+r^{2}} p+\left|\frac{z q^{\prime}(z)}{q(z)}\right| \\
\leq & -\frac{1-r^{2}}{1+r^{2}} p+\frac{2 r}{1-r^{2}}=\frac{-p r^{4}+2 r^{3}+2 p r^{2}+2 r-p}{1-r^{4}} .
\end{aligned}
$$

It is easy to know that if $-p r^{4}+2 r^{3}+2 p r^{2}+2 r-p<0$, we have $\mathbb{R}\left(1+\frac{z f^{\prime \prime}(z)}{f^{\prime}(z)}\right)<$ 0 . Let

$$
T_{p}(r)=-p r^{4}+2 r^{3}+2 p r^{2}+2 r-p<0,
$$


because $T_{p}(0)=-p<0, T_{p}(1)=4$, and

$$
T_{p}^{\prime}(r)=-4 p r^{3}+6 r^{2}+4 p r+2=4 p r\left(1-r^{2}\right)+6 r^{2}+2>0(0<r<1) .
$$

It follows that $T_{p}(r)$ are strictly monotone increasing functions of $r$, and for very $p$, equation $T_{p}(r)=0$ has only a root $r_{p}$ in interval $(0,1)$, solve those equations we get the $r_{p}$ in (4.3). Thus when $0<|z|<r_{p}, \mathbb{R}\left(1+\frac{z f^{\prime \prime}(z)}{f^{\prime}(z)}\right)<0$, that is, $f(z)$ is meromophic convex in $0<|z|<r_{p}$.

\section{REFERENCES}

[1] M. Aouf and H. Srivastava, "A new criterion for meromorphically p-valent convex functions of order alpha," Math. Sci. Res. Hot-Line 1, vol. 8, pp. 7-12, 1997.

[2] K. Bogumila, L. Adam, and H. Srivastava, "A note on the Fekete-Szego problem for close-toconvex functions with respect to convex functions," Publ. Inst. Math., vol. 115, pp. 143-149, 2017, doi: 10.2298/PIM1715143K.

[3] C. Gao and S. Zhou, "on a class of analytic fuctions related to the starlike functions," Kyungpook. Math., vol. 45, pp. 123-130, 2005.

[4] A. Goodman, Univalent Functions. Washington: Polygonal Publishing House, 1983.

[5] H. Guney, G. Murugusundaramoorthy, and H. Srivastava, "The Second Hankel Determinant for a Certain Class of Bi-Close-to-Convex Functions," Results Math, vol. 74, no. 93, pp. 1-13, 2019, doi: 10.1007/s00025-019-1020-0.

[6] J. Jahangiri, Y. Kim, and H. Srivastava, "Construction of a Certain Class of Harmonic Close-ToConvex Functions Associated with the Alexander Integral Transform," Integral Transforms Spec. Funct., vol. 14, pp. 237-242, 2003, doi: 10.1080/1065246031000074380.

[7] J. Kowalczyk and E. Bomba, "On a subclass of close-to-convex functions," Appl. Math. Lett., vol. 23, pp. 1147-1151, 2010, doi: 10.1016/j.aml.2010.03.004.

[8] J. Liu and H. Srivastava, "Some convolution conditions for starlikeness and convexity of meromorphically multivalent functions," Appl. Math. Lett., vol. 16, pp. 13-16, 2003, doi: 10.1016/S0893-9659(02)00138-6.

[9] J. Liu and H. Srivastava, "Classes of meromorphically multivalent functions associated with the generalized hypergeometric function," Math. Comput., vol. 39, pp. 21-34, 2004, doi: 10.1016/S0895-7177(04)90503-1.

[10] A. Livingston, "P-valent close-to-convex functions," Trans.Amer.Math.Soc., vol. 115, pp. 161179,1965 .

[11] S. Mahmood, J. Sokol, H. Srivastava, and S. Malik, "Some Reciprocal Classes of Close-toConvex and Quasi-Convex Analytic Functions," Mathematics, vol. 7, no. 309, pp. 1-13, 2019, doi: 10.3390/math7040309.

[12] S. Owa, M. Nunokawa, H. Saitoh, and H. Srivastava, "Close-to-convexity, starlikeness, and convexity of certain analytic functions," Appl. Math. Lett., vol. 15, pp. 63-69, 2002, doi: 10.1016/S0893-9659(01)00094-5.

[13] V. Ravichandran, S. Kumar, and K. Subramanian, "Convolution conditions for spirallikeness and convex spirallikeness of certain meromorphic p-valent functions," J. Inequal.Pure Appl.Math, vol. 5, pp. 1-7, 2004.

[14] W. Royster, "Meromorrphic Starlike Multivalent Functions," J. Funct. Anal, vol. 202, pp. 67-97, 2003. 
[15] H. Srivastava, Q. Ahmad, M. Darus, N. Khan, B. Khan, N. Zaman, and H. Shah, "Upper Bound of the Third Hankel Determinant for a Subclass of Close-to-Convex Functions Associated with the Lemniscate of Bernoulli," Math., vol. 7, no. 848, pp. 1-10, 2019, doi: 10.3390/math7090848.

[16] H. Srivastava and D. Bansal, "Close-to-convexity of a certain family of q-Mittag-Leffler functions," J. Nonlinear Var. Anal., vol. 1, pp. 61-69, 2017.

[17] H. Srivastava, M. Khan, and M. Arif, “ Some subclasses of close-to-convex mappings associated with conic regions," Appl. Math. Comput., vol. 285, pp. 94-102, 2016, doi: 10.1016/j.amc.2016.03.025.

[18] H. Srivastava, N. Khan, M. Darus, M. Rahim, Q. Ahmad, and Y. Zeb, "Properties of Spiral-Like Close-to-Convex Functions Associated with Conic Domains," Mathematics, vol. 7, no. 706, pp. 1-12, 2019, doi: 10.3390/math7080706.

[19] H. Srivastava, S. Li, and H. Tang, "Certain classes of $k$-uniformly close-to-convex functions and other related functions defined by using the Dziok-Srivastava operator," Bull. Math. Anal. Appl., vol. 1, pp. 49-63, 2009.

[20] H. Srivastava, A. Mishra, and M. Das, "The Fekete-Szego problem for a subclass of closeto-convex functions," Complex Variables Theory Appl., vol. 69, pp. 145-163, 2001, doi: 10.1080/17476930108815351.

[21] Z. Wang and D. Chen, "On a subclass of close-to-convex functions," Hacet. J. Math. Stat., vol. 38, pp. 95-101, 2009, doi: 10.1016/j.aml.2010.03.004.

[22] Z. Wang, Y. Sun, and N. Xu, "Some Properties of Certain Meromorphic Close-to-convex Functions," Applied Mathematics Letters, vol. 25, pp. 454-460, 2012, doi: 10.1016/j.aml.2011.09.035.

[23] Z. Wang, Y. Sun, and Z. Zhang, "Certain classes of meromorphic multivalent functions ," Comput. Math. Appl., vol. 58, pp. 1408-1417, 2009, doi: 10.1016/j.camwa.2009.07.020.

[24] Q. Xu, H. Srivastava, and Z. Li, "A certain subclass of analytic and close-to-convex functions," Appl. Math. Lett., vol. 24, pp. 396-401, 2011, doi: 10.1016/j.aml.2010.10.037.

[25] S. Yuan, Z. Liu, and H. Srivastava, "Some inclusion relationships and integral-preserving properties of certain subclasses of meromorphic functions associated with a family of integral operators," J. Math. Anal. Appl, vol. 337, pp. 505-515, 2008, doi: 10.1016/j.jmaa.2007.04.018.

\section{Author's address}

\section{Hong-Guang $\mathbf{L i}$}

Huai Hua College, Department of Mathematics, HuaiHua, China

E-mail address: Ihg20052008@126.com 